\title{
Nonlinear damping in graphene resonators
}

\author{
Alexander Croy, ${ }^{*}$ Daniel Midtvedt, Andreas Isacsson, and Jari M. Kinaret \\ Department of Applied Physics, Chalmers University of Technology, S-412 96 Göteborg, Sweden \\ (Received 5 April 2012; revised manuscript received 12 October 2012; published 20 December 2012)
}

\begin{abstract}
Based on a continuum mechanical model for single-layer graphene, we propose and analyze a microscopic mechanism for dissipation in nanoelectromechanical graphene resonators. We find that coupling between flexural modes and in-plane phonons leads to linear and nonlinear damping of out-of-plane vibrations. By tuning external parameters such as bias and ac voltages, one can cross over from a linear- to a nonlinear-damping dominated regime. We discuss the behavior of the effective quality factor in this context.
\end{abstract}

DOI: 10.1103/PhysRevB.86.235435

\section{INTRODUCTION}

Advances in fabrication and detection techniques have enabled a wide range of experimental realizations of carbonbased nanoelectromechanical (NEM) resonators. ${ }^{1-4}$ However, to optimize their operation, an increased understanding of dissipation mechanisms is needed. For NEM resonators in general, several processes leading to linear damping (LD) have been investigated. ${ }^{5-8}$ Specifically, for graphene, at high temperatures, Ohmic losses in the metallic gate and the graphene sheet have been argued to limit the quality factor. ${ }^{9}$ Recently, the focus has shifted to study quantum aspects of mechanical motion, ${ }^{10,11}$ such as mechanical cat states, ${ }^{12}$ which require a more detailed understanding of dissipation and decoherence mechanisms.

Since graphene-based resonators exhibit nonlinear behavior, one can expect the damping also to be amplitude dependent. ${ }^{13-15}$ Nonlinear damping (NLD) was reported in recent experiments on graphene and carbon nanotube resonators. ${ }^{4}$ However, little is known about the underlying physical mechanism, and typically phenomenological models are employed. ${ }^{13-15}$ In these models, the resonator is coupled to a bath of harmonic oscillators. For couplings that depend quadratically on the resonator amplitude, it is known that NLD emerges. ${ }^{13,16,17}$

For carbon-based resonators, such a coupling naturally arises if the strain couples linearly to the degrees of freedom of some subsystem, which can be regarded as a bath. Two examples are the interaction between phonons and electrons ${ }^{18,19}$ and the coupling of mechanical modes. The relative importance of the two mechanisms is a priori not known and will also depend on the details of the experimental realization.

In order to quantify the importance of the mechanical dissipation channel for NLD, we analyze the coupling between flexural modes and in-plane phonons. We show that it leads to a quadratic coupling and, consequently, to both LD and NLD. Whether LD or NLD dominates is determined by the ratio of vibrational amplitude and static deflection. We give an estimate for the expected crossover between LD and NLD, which can be experimentally verified.

\section{MODEL AND METHOD}

We consider a graphene sheet of length $L$ and breadth $b$, suspended over a trench of width $\ell$ (cf. Fig. 1). The van der Waals attraction between the graphene and the substrate clamps down the sheet outside the suspended region. ${ }^{20-22}$
The trench is modeled by allowing the sheet to freely displace vertically in this region. Since out-of-plane displacement is accompanied by in-plane stretching or compression, flexural motion is converted into in-plane phonons in the suspended region. The clamping constrains the out-ofplane motion over the substrate, but still allows for small inplane displacements. Consequently, in-plane phonons created in the suspended region transport energy away from this region. In contrast to a phenomenological modeling approach, we can relate dissipation to specific properties of the substrate and the graphene-substrate coupling. These properties can be obtained independently by theoretical or experimental means.

The dynamics of graphene NEM resonators are well described by the continuum theory of two-dimensional (2D) membranes. ${ }^{23}$ For a resonator made from a sheet lying in the $x y$ plane, this theory is conveniently formulated in terms of the in-plane displacement fields $u(x, y), v(x, y)$ in the $x$ and $y$ directions, respectively, and the displacement field in the $z$ direction, $w(x, y)$. The equations of motion follow from the free energy $\mathcal{F}=\int d x d y\left[\mathcal{F}_{b}+\mathcal{F}_{s}\right]$ where $\mathcal{F}_{b}=\frac{\kappa}{2}|\Delta w|^{2}$ is the free energy density associated with pure bending and $\mathcal{F}_{s}=$ $\frac{1}{2} \sum_{i, j} \sigma_{i j} \epsilon_{i j}$ is associated with stretching of the membrane. The symmetric 2D strain and stress tensors are here defined as

$$
\begin{aligned}
\epsilon_{x x} & =u_{, x}+w_{, x}^{2} / 2, \quad 2 \epsilon_{x y}=\left(u_{, y}+v_{, x}\right)+w_{, x} w_{, y}, \\
\epsilon_{y y} & =v_{, y}+w_{, y}^{2} / 2,
\end{aligned}
$$

and

$$
\begin{aligned}
& \sigma_{x x}=\left(\lambda_{\mathrm{G}}+2 \mu_{\mathrm{G}}\right) \epsilon_{x x}+\lambda_{\mathrm{G}} \epsilon_{y y}, \quad \sigma_{x y}=2 \mu_{\mathrm{G}} \epsilon_{x y}, \\
& \sigma_{y y}=\left(\lambda_{\mathrm{G}}+2 \mu_{\mathrm{G}}\right) \epsilon_{y y}+\lambda_{\mathrm{G}} \epsilon_{x x},
\end{aligned}
$$

respectively. Spatial derivatives are denoted by subscripts, i.e., $u_{, x}=\partial u / \partial x$. The expression for the free energy, which is similar to that for large deflections of a plate, ${ }^{24}$ contains three material parameters, the bending energy $\kappa \approx 1.1-1.6 \mathrm{eV}$, and the Lamé parameters $\mu_{\mathrm{G}} \approx 146 \mathrm{~N} / \mathrm{m}$ and $\lambda_{\mathrm{G}} \approx 48 \mathrm{~N} / \mathrm{m}$ for graphene. ${ }^{25-28}$ To study qualitatively the effect of phonon radiation into the supporting substrate, we assume for simplicity a quasi-one-dimensional (1D) situation where variations in the $y$ direction are disregarded. This would be valid for a wide sheet where deviations from this assumption are confined to the regions around the edges. In this case, we have only the displacement fields $u(x, t)$ and $w(x, t)$. In any realistic functioning device, there is some small amount of built-in strain. In practice, this implies that the energy contribution from the bending energy is always negligible for the 


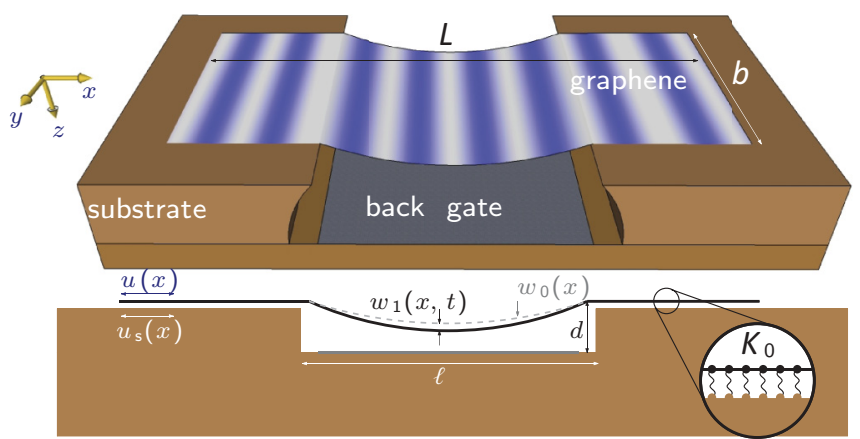

FIG. 1. (Color online) Schematic view of a suspended graphene membrane over a trench in an insulating substrate. A metallic gate is used for actuating the resonator. In-plane phonons are created in the suspended region and dissipate energy as they propagate away.

lowest-lying flexural modes. ${ }^{27}$ Hence, to a good approximation we have for the quasi-1D graphene resonator attached to a substrate the free energy density

$$
\begin{aligned}
\mathcal{F}(x, y)= & \frac{T_{1}}{2}\left(u_{, x}^{2}+u_{, x} w_{, x}^{2}+\frac{1}{4} w_{, x}^{4}\right) \\
& +\frac{1}{2} K(x)\left(u-u_{\mathrm{S}}\right)^{2}+\mathcal{E}_{\text {ext }}[w],
\end{aligned}
$$

where we have defined $T_{1}=\lambda_{\mathrm{G}}+2 \mu_{\mathrm{G}}$. The potential $\mathcal{E}_{\mathrm{ext}}[w]$ accounts for interactions used to actuate the resonator. The second to last term couples the graphene displacement to the substrate displacement $u_{\mathrm{S}}(x, y)$ in a harmonic approximation, ${ }^{29}$ which largely allows us to obtain an analytical description.

The function $K(x)$ restricts this coupling to the supported region, i.e., $K(x)=K_{0} \Theta(|x|-\ell / 2)$ with $\Theta$ being the Heaviside step function. The substrate is modeled as an elastic half-space and displacement at the surface $\vec{s}(\vec{x}, z=0, t)=$ $\left(u_{\mathrm{S}}, v_{\mathrm{S}}, w_{\mathrm{S}}\right)$ is given in terms of a response function ${ }^{24,30,31}$

$$
s_{\mu}(\vec{x}, z=0, \omega)=-\sum_{\nu} \int \frac{d^{2} x^{\prime}}{(2 \pi)^{2}} R_{\mu \nu}\left(\vec{x}-\vec{x}^{\prime}, \omega\right) \sigma_{\nu z}\left(\vec{x}^{\prime}, \omega\right) .
$$

Consistent with the 1D model of the graphene sheet, only $\bar{u}_{\mathrm{S}}(x) \equiv \int_{-b / 2}^{b / 2} d y u_{\mathrm{S}}(x, y)$ is considered. Within the harmonic approximation, $\sigma_{x z}=K(x)\left(u-u_{S}\right)$.

The free energy (2) leads to a coupling between flexural vibrations and in-plane motion via the coupling energy $\mathcal{E}_{\text {coup }}=$ $\left(T_{1} / 2\right) u_{, x} w_{, x}^{2}$, which is nonlinear in the flexural vibration amplitude. This coupling leads to NLD of the flexural vibrations. $^{13,15-17}$

\section{A. Equations of motion}

The equations of motion for the out-of-plane and in-plane vibrations resulting from Eq. (2) are

$$
\begin{aligned}
\rho_{\mathrm{G}} \ddot{w}-\frac{T_{1}}{2} \frac{d}{d x}\left(2 u_{, x} w_{, x}+w_{, x}^{3}\right) & =f_{\mathrm{dc}}+f_{\mathrm{ac}} \cos (\Omega t), \\
\rho_{\mathrm{G}} \ddot{u}-\frac{T_{1}}{2} \frac{d}{d x}\left(2 u_{, x}+w_{, x}^{2}\right) & =-K(x)\left(u-\bar{u}_{\mathrm{S}} / b\right),
\end{aligned}
$$

where $f_{\mathrm{dc}}(x)$ and $f_{\mathrm{ac}}(x) \cos (\Omega t)$ are the static and timedependent parts of the actuation force. Typically, electrostatic actuation is used, resulting from a time-dependent back-gate voltage of the form $V_{\mathrm{bg}}(t)=V_{\mathrm{dc}}+V_{\mathrm{ac}} \cos (\Omega t)$ with $V_{\mathrm{dc}} \gg$ $V_{\mathrm{ac}}$. To simplify the analysis, we assume the equilibrium stress field resulting from $f_{\mathrm{dc}}$ to be spatially uniform and equal to the tensile stress $T_{0}$ on the boundary. ${ }^{24}$ Generally, at a given back-gate bias voltage, the resonance frequency $\Omega_{0}\left(V_{\mathrm{dc}}\right)$ depends on initial stress and contains a shift due to electrostatic forces. This so-called tuning behavior will be further discussed in Sec. III A.

Since Eq. (4b) is linear in $u$, the influence of the environment can be accounted for by a Green's function embedding technique. The solution

$u(x, t)=\int d x^{\prime} \int d t^{\prime} G\left(x, x^{\prime}, t-t^{\prime}\right) \frac{c^{2}}{2} \frac{d}{d x^{\prime}} w_{, x^{\prime}}{ }^{2}\left(x^{\prime}, t^{\prime}\right)$

is given in terms of the in-plane response function $G$, which contains information about the attachment to the substrate via Eq. (3). The speed of sound in graphene is denoted by $c=$ $\sqrt{T_{1} / \rho_{\mathrm{G}}}$, where $\rho_{\mathrm{G}}$ is the mass density of graphene.

\section{B. Flexural mode dynamics}

Next, we consider the fundamental flexural mode and set $w(x, t)=q(t) \phi(x)$ for $|x| \leqslant \ell / 2$ and zero otherwise. The mode shape $\phi$ is normalized to the length of the resonator. Upon projecting Eq. (4a) onto the fundamental mode, an ordinary differential equation for the vibration amplitude $q$ is obtained. Further, moving to a rotating frame, we write $q(t)=\left\{q_{0}+\frac{1}{2}\left[q_{1}(t) e^{i \Omega t}+q_{1}^{*}(t) e^{-i \Omega t}\right]\right\}$ and $\dot{q}(t)=\frac{i \Omega}{2}\left[q_{1}(t) e^{i \Omega t}-q_{1}^{*}(t) e^{-i \Omega t}\right]$. Inserting these expressions into the equation of motion and performing the averaging yields an equation for the slowly varying amplitude $q_{1}$ [13], which contains memory terms related to linear and nonlinear damping. As the time scales for flexural motion and in-plane phonons are well separated $\left(\Omega_{0} \ll c / \ell\right)$, the memory terms can be eliminated. This procedure corresponds to a Markov approximation. ${ }^{13}$ It is convenient to define new quantities

$\hat{\chi}(\Omega)=\frac{c^{2}}{2} \int_{-l / 2}^{l / 2} d x \int_{-l / 2}^{l / 2} d x^{\prime} \frac{d}{d x}\left[\phi_{, x}^{2} \hat{G}\left(x, x^{\prime},-\Omega\right)\right] \frac{d}{d x^{\prime}} \phi_{, x^{\prime}}^{2}$,

where $\hat{G}\left(x, x^{\prime}, \omega\right)=(2 \pi)^{-1} \int d \tau G\left(x, x^{\prime}, \tau\right) e^{i \omega \tau}$ is the Fourier transform of the in-plane response function.

We obtain an equation of motion for the complex envelope function

$$
\begin{aligned}
m \dot{q}_{1}= & {\left[i m\left(\Omega_{0}-\Omega\right) q_{1}+i \frac{3}{8} \frac{\alpha}{\Omega_{0}}\left|q_{1}\right|^{2} q_{1}\right.} \\
& \left.-\frac{1}{2} \gamma q_{1}-\frac{1}{8} \eta\left|q_{1}\right|^{2} q_{1}-\frac{i}{2 \Omega_{0}} g\right] .
\end{aligned}
$$

For finite temperatures, this equation has to be supplemented by noise forces, satisfying the fluctuation-dissipation relations. The thermally induced vibrations can lead to an additional broadening of the response curves. ${ }^{13,32}$ In order to obtain a lower bound of LD and NLD, we will work in the limit of zero temperature. In Eq. (7), the coefficients $m=\rho_{\mathrm{G}} \ell b, \alpha, \gamma$, and $\eta$ denote the suspended mass, the Duffing elastic constant, 
linear and nonlinear damping, respectively. They are given in terms of $\hat{\chi}$ as follows:

$$
\begin{aligned}
\alpha & =\alpha_{0}-\frac{T_{1} b}{2} \frac{4}{3} \operatorname{Re}\left(\hat{\chi}(0)+\frac{1}{2} \hat{\chi}(2 \Omega)\right), \\
\gamma & =-\frac{T_{1} b}{2 \Omega_{0}} q_{0}^{2} 4 \operatorname{Im} \hat{\chi}(\Omega), \\
\eta & =-\frac{T_{1} b}{2 \Omega_{0}} 2 \operatorname{Im} \hat{\chi}(2 \Omega) .
\end{aligned}
$$

Here, the bare Duffing constant is given by $\alpha_{0}=\left(T_{1} b / 2\right)$ $\int d x \phi_{, x}(x)^{4}$. The driving strength is $g=\int d x \phi(x) f_{\mathrm{ac}}(x)$. In accordance with our previous simplifications, we neglect the small polaronic shift of $\Omega_{0}$, which is proportional to $\operatorname{Re} \hat{\chi}$, and an additional shift of $\alpha$ due to the broken symmetry in the presence of static deflection. Equation (7) is similar to the equations used to model NLD in micromechanical resonators ${ }^{14,15}$ and recent experiments on carbon-based resonators, ${ }^{4}$ the difference being the dependence of the damping coefficients in Eq. (8) on the driving frequency.

In Eq. (7), the prevailing damping mechanism is determined by the ratio

$$
\tilde{\delta} \equiv \frac{\eta\left|q_{1}\right|^{2}}{4 \gamma} \approx \frac{\operatorname{Im} \hat{\chi}(2 \Omega)}{8 \operatorname{Im} \hat{\chi}(\Omega)} \frac{\left|q_{1}^{\max }\right|^{2}}{q_{0}^{2}} .
$$

Here, $\left|q_{1}^{\max }\right|$ denotes the maximum amplitude of the response for a given driving strength. Thus, $\tilde{\delta}$ is determined by the ratio of the overlap integrals defined in Eq. (6), which are purely geometrical quantities, and the ratio between the vibrational amplitude and the static deflection. For a small static deflection, it is therefore expected that NLD dominates the damping caused by phonon radiation. Similarly, the dimensionless ratio

$$
\tilde{\eta}=\frac{\eta \Omega_{0}}{\alpha}
$$

measures the relative importance of the two nonlinearities in Eq. (7). ${ }^{14}$ For $\tilde{\eta}<\sqrt{3}$, the well-known bifurcation of the Duffing equation is present, while for $\tilde{\eta}>\sqrt{3}$ this bifurcation vanishes. The ratio $\tilde{\eta}$ is also a purely geometrical factor, apart from the weak dependence of $\Omega_{0}$ on the static deformation of the graphene.

\section{Numerical method}

To compute the overlap integrals (6), we first consider the Fourier-transformed response of the substrate (3):

$$
\begin{aligned}
\bar{u}_{\mathrm{S}}(x, \omega)= & -\int_{-L / 2}^{L / 2} \frac{d x^{\prime}}{(2 \pi)^{2}} \int_{-b / 2}^{b / 2} d y^{\prime} \int_{-b / 2}^{b / 2} d y \\
& \times R_{x x}\left(x-x^{\prime}, y-y^{\prime}, \omega\right) \sigma_{x z}\left(x^{\prime}, y^{\prime}, \omega\right) \\
\approx & -\int_{-L / 2}^{L / 2} \frac{d x^{\prime}}{(2 \pi)^{2}} \bar{R}_{x x}\left(x-x^{\prime}, \omega\right) \bar{\sigma}_{x z}\left(x^{\prime}, \omega\right) .
\end{aligned}
$$

In the second step, in order to get a purely 1D response function, we have approximated the $y^{\prime}$ dependence of $\sigma_{x z}\left(x^{\prime}, y^{\prime}\right)$ by the mean value $\frac{1}{b} \bar{\sigma}_{x z}$ and defined $\bar{R}_{x x}\left(x-x^{\prime}, \omega\right) \equiv \frac{1}{b} \int_{-b / 2}^{b / 2} d y^{\prime} \int_{-b / 2}^{b / 2} d y R_{x x}\left(x-x^{\prime}, y-y^{\prime}, \omega\right) .{ }^{33}$ The response function $R_{\mu \nu}$ for an elastic half-space is known analytically $24,30,31$ and mainly depends on the longitudinal and transversal sound velocities of the substrate (see Appendix A).

Evaluating Eq. (11) at discrete positions $\left\{x_{i}\right\}_{1}^{N}$ leads to the linear system

$$
\mathbb{K} \mathbf{u}_{\mathrm{S}}(\omega)=-[\mathbb{I}-\mathbb{K} \mathbb{R}(\omega)]^{-1} \mathbb{K} \mathbb{R}(\omega) \mathbb{K} \mathbf{u}(\omega),
$$

which can be solved for $\bar{u}_{\mathrm{S}}\left(x_{i}, \omega\right)$. Here, boldface symbols denote vectors of length $N$, e.g., $\mathbf{u}=\left[u\left(x_{1}\right), \ldots, u\left(x_{N}\right)\right]$ and open face symbols are $N \times N$ matrices. In particular, $\mathbb{I}_{i j}=\delta_{i, j}, \mathbb{K}_{i j}=K\left(x_{i}\right) \delta_{i, j}$, and $\mathbb{R}_{i j}=(2 \pi)^{-2} \bar{R}_{x x}\left(x_{i}-x_{j}, \omega\right)$. Using this result and the discretized version of the equation of motion (4b), one obtains an equation for the in-plane response function $\mathbb{G}_{i j}=\hat{G}\left(x_{i}, x_{j}, \omega\right)$ :

$$
\left[-\omega^{2} \mathbb{I}-c^{2} \mathbb{L}+\frac{1}{\rho_{\mathrm{G}}}[\mathbb{I}-\mathbb{K} \mathbb{R}(\omega)]^{-1} \mathbb{K}\right] \mathbb{G}(\omega)=\mathbb{I},
$$

where $\mathbb{L}$ is the discrete second derivative. ${ }^{34}$ Approximating the integrations in Eq. (6) by numerical quadratures, one finally obtains

$$
\hat{\chi}(\Omega)=\frac{c^{2}}{2} \boldsymbol{\Phi}^{\mathrm{t}} \mathbb{G}(-\Omega) \boldsymbol{\Phi}
$$

with $\boldsymbol{\Phi}_{i}=\left.\frac{d}{d x} \phi_{, x}^{2}\right|_{x=x_{i}}$, which allows the computation of $\hat{\chi}$ for a given geometry. The parameters entering the equation of motion can then be calculated using Eqs. (8). Following Ref. 14 , we set $\tilde{\gamma}=\gamma /\left(m \Omega_{0}\right), \tilde{\eta}=\eta \Omega_{0} / \alpha, \tilde{g}=g \sqrt{\frac{\alpha}{m^{3}}} / \Omega_{0}^{3}$, $\tilde{\Omega}=\Omega / \Omega_{0}$, and $\tilde{q}=q \sqrt{\alpha / m \Omega_{0}^{2}}$. In the limit of weak LD, $\tilde{\gamma} \ll 1$, the response of the resonator is determined solely by the dimensionless parameters $\tilde{\eta}, \tilde{g}$, and $\tilde{\Omega}$, describing the nonlinear damping, the driving strength, and the driving frequency.

\section{RESULTS}

To quantify the influence of LD and NLD, we consider the setup shown in Fig. 1 with a back-gate voltage $V_{\mathrm{bg}}=$ $V_{\mathrm{dc}}+V_{\mathrm{ac}} \cos (\Omega t)$. The fundamental-mode shape is taken to be $\phi(x)=\sqrt{2} \cos (\pi x / \ell)$, which gives $\alpha_{0}=3 T_{1} \pi^{4} b /\left(4 \ell^{3}\right)$. Within a parallel plate model for electrostatic actuation, the force acting on the graphene sheet is given by

$$
\begin{aligned}
f(x) & =\frac{\partial}{\partial w} \frac{1}{2} C(w) V_{\mathrm{bg}}^{2} \\
& \approx-\frac{\epsilon_{0}}{2[d+q(t) \phi(x)]^{2}}\left[V_{\mathrm{dc}}^{2}+2 V_{\mathrm{dc}} V_{\mathrm{ac}} \cos (\Omega t)\right],
\end{aligned}
$$

where $C(w)=\epsilon_{0} /(d+w)$ is the capacitance of a parallel plate capacitor with plates being separated by the distance $d+w$ and $\epsilon_{0}$ is the vacuum permittivity. The distance is determined by the depth $d$ of the trench and the flexural displacement $w$ of the resonator. In the second line, we further assumed $V_{\mathrm{dc}} \gg V_{\mathrm{ac}}$, which is typically found in experiments. The force can be separated into a static and a time-dependent part $f=f_{\mathrm{dc}}+f_{\mathrm{ac}} \cos (\Omega t)$ with $f_{\mathrm{dc}} \propto V_{\mathrm{dc}}^{2}$ and $f_{\mathrm{ac}} \propto V_{\mathrm{dc}} V_{\mathrm{ac}}$, respectively. Since the displacement, which is on the order of a few nanometers, is much smaller than the trench depth, the force can be expanded in powers of $w$. Accordingly, the driving strength in Eq. (7) becomes $g=2 \sqrt{2} \ell b \epsilon_{0} V_{\mathrm{dc}} V_{\mathrm{ac}} /\left(\pi d^{2}\right)$. Moreover, the static displacement can be found by solving Eqs. (4a) and (4b) in the static limit (see Appendix B). This yields $q_{0} \approx \sqrt{2} \ell^{2} \epsilon_{0} V_{\mathrm{dc}}^{2} /\left(\pi^{3} d^{2} T_{0}\right)$. Note the dependence on the 
TABLE I. Graphene and resonator parameters used for the calculations in Figs. 3 and 4. Graphene and substrate parameters are taken from Refs. 35 and 36.

\begin{tabular}{lcc}
\hline \multicolumn{3}{c}{ Graphene and substrate parameters } \\
\hline Graphene mass density & $\rho_{\mathrm{G}}$ & $7.6 \times 10^{-7} \mathrm{~kg} \mathrm{~m}^{-2}$ \\
$\lambda_{\mathrm{G}}+2 \mu_{\mathrm{G}}$ & $T_{1}$ & $340 \mathrm{~N} \mathrm{~m}^{-1}$ \\
$\mathrm{SiO}_{2}$ mass density & $\rho_{\mathrm{S}}$ & $2.2 \times 10^{3} \mathrm{~kg} \mathrm{~m}^{-3}$ \\
$\mathrm{SiO}_{2}$ sound velocities & $c_{\mathrm{L}} / c$ & 0.28 \\
& $c_{\mathrm{T}} / c$ & 0.18 \\
Coupling strength & $K_{0}$ & $1.82 \times 10^{20} \mathrm{~N} \mathrm{~m}^{-3}$ \\
& Resonator parameters & \\
Total length & $L$ & $2 \mu \mathrm{m}$ \\
Length & $\ell$ & $1 \mu \mathrm{m}$ \\
Width & $b$ & $1 \mu \mathrm{m}$ \\
Distance to gate & $d$ & $330 \mathrm{~nm}$ \\
Tensile stress & $T_{0}$ & $0.34 \mathrm{~N} \mathrm{~m}^{-1}$ \\
\hline \hline
\end{tabular}

tensile stress $T_{0} ; q_{0}$ becomes smaller for increasing tensile stress.

In the following, we consider a graphene resonator with dimensions and parameters as given in Table I. We checked that the results do not change for larger values of the total length $L$. Using Eqs. (8) and (10), we obtain $\alpha / \alpha_{0} \approx 0.64$ and $\tilde{\eta} \approx$ $7 \times 10^{-4}$. The latter implies bistable behavior of the resonator. In general, these values depend sensitively on the geometry of the graphene sheet and on the substrate. Our results provide a "best-case" estimate since the substrate is treated as a semiinfinite medium and the trench is modeled by the positiondependent coupling $K(x)$. Lifting these restrictions will lead to a stronger response of the substrate, and more dissipation.

\section{A. Resonance frequency}

As described in Sec. II A, the resonance frequency $\Omega_{0}\left(V_{\mathrm{dc}}\right)$ depends on the initial stress and the bias voltage. The dependence of $\Omega_{0}$ on bias voltage, the so-called tuning curve, is a characteristic feature of NEMS devices. It is a result of the competition between softening (decreasing $\Omega_{0}$ ) due to the electrostatic force [Eq. (15)], and stiffening (increasing $\Omega_{0}$ ) due to the Duffing nonlinearity of the graphene sheet.

To obtain the tuning curve, we separate static and dynamic contributions to the displacement fields

$$
\begin{aligned}
& w(x, t)=w_{0}(x)+\delta w(x, t), \\
& u(x, t)=u_{0}(x)+\delta u(x, t)
\end{aligned}
$$

and insert these expressions into the equations of motion given by Eqs. (4). The static solutions $w_{0}$ and $u_{0}$ are calculated in Appendix B. Further, we expand the static force $f_{\mathrm{dc}}(x)$ up to first order in $\delta w$ :

$$
f_{\mathrm{dc}} \approx-\frac{\epsilon_{0} V_{\mathrm{dc}}^{2}}{2\left(d+w_{0}\right)^{2}}+\frac{\epsilon_{0} V_{\mathrm{dc}}^{2}}{\left(d+w_{0}\right)^{3}} \delta w .
$$

The resonance frequency is then obtained by collecting terms, which are linear in the vibration amplitude $\delta w$. There are three such terms, which contribute to the resonance frequency:

$$
\Omega_{0}^{2}\left(V_{\mathrm{dc}}\right)=\Omega_{0}^{2}(0)+\Delta \Omega_{\text {mech. }}^{2}-\Delta \Omega_{\mathrm{el} .}^{2}
$$

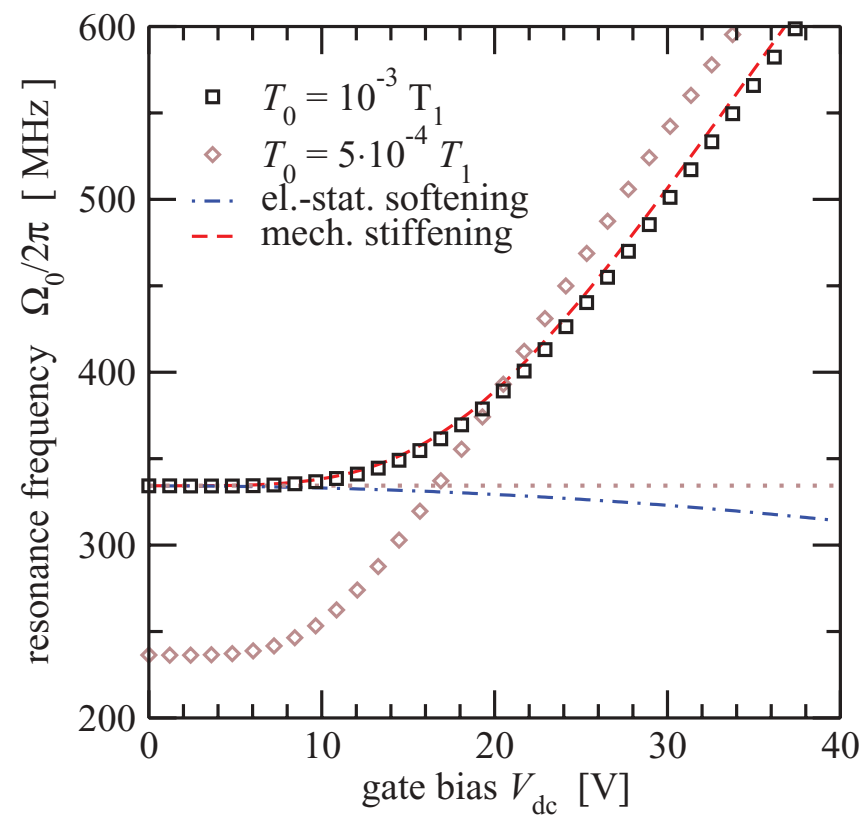

FIG. 2. (Color online) Resonance frequency $\Omega_{0}$ vs bias voltage. Symbols denote results of numerical calculation. The dashed (red) and dashed-dotted (blue) lines show the contributions of mechanical stiffening and electrostatic softening for $T_{0}=10^{-3} T_{1}$, respectively. Parameters are given in Table I.

with

$$
\begin{aligned}
\Omega_{0}^{2}(0) & =\frac{T_{0}}{\rho_{\mathrm{G}}} \frac{\pi^{2}}{\ell^{2}}, \\
\Delta \Omega_{\text {mech. }}^{2}\left(V_{\mathrm{dc}}\right) & =2 \frac{T_{1} \pi^{4}}{\rho_{\mathrm{G}} \ell^{4}} q_{0}^{2}=\frac{8}{3 m} \alpha_{0} q_{0}^{2}, \\
\Delta \Omega_{\mathrm{el} .}^{2}\left(V_{\mathrm{dc}}\right) & =\frac{\epsilon_{0} V_{\mathrm{dc}}^{2}}{d^{3} \rho_{\mathrm{G}}} .
\end{aligned}
$$

The three contributions are due to initial strain, mechanical stiffening, and electrostatic softening, respectively. Since the static deflection $q_{0}$ depends on the bias voltage $V_{\mathrm{dc}}$, the last two terms yield the voltage-dependent tuning behavior.

Figure 2 shows the tuning curve for the parameters given in in Table I. For voltages $V_{\mathrm{dc}}>10 \mathrm{~V}$, the resonance frequency (squared) is mainly determined by the mechanical stiffening, which scales with $V_{\mathrm{dc}}^{4}$, while the softening term scales with $V_{\mathrm{dc}}^{2}$ according to Eqs. (18).

Depending on the specific geometry and the initial stress, the resonance frequency of the resonator may be substantially tuned using the bias voltage. Since the linear and nonlinear damping coefficients given by Eqs. (8) depend on frequency, the magnitude of LD and NLD will, in principle, also be influenced by the tuning curve. In order to disentangle the influence of $\Omega_{0}\left(V_{\mathrm{dc}}\right)$ and the coupling to the in-plane phonons, we will only consider a constant resonance frequency $\Omega_{0}=\Omega_{0}(0)=$ $\sqrt{T_{0} / \rho_{\mathrm{G}}}(\pi / \ell)$ in the following discussions (see Appendix $\mathrm{C}$ for the influence of the tuning on the quality factor).

\section{B. Damping ratio}

The relative importance of LD and NLD, which is quantified by $\tilde{\delta}$ defined in Eq. (9), is determined by the ratios 
$\operatorname{Im} \hat{\chi}(2 \Omega) /[8 \operatorname{Im} \hat{\chi}(\Omega)]$ and $\left|q_{1}^{\max }\right| / q_{0}$. The former weakly depends on the geometric details.

For small $\Omega$, one can expand $\operatorname{Im} \hat{\chi}(\Omega)$ in odd powers of $\Omega$. As $\operatorname{Im} \hat{\chi}$ is proportional to the density of states of the substrate phonons, $D(\Omega) \propto \Omega$, we expect on symmetry grounds for a quasi-1D geometry that $\operatorname{Im} \hat{\chi}(\Omega) \propto \Omega^{3}$. Consistent with this expectation, we obtain numerically $\operatorname{Im} \hat{\chi}(2 \Omega) /[8 \operatorname{Im} \hat{\chi}(\Omega)] \approx 0.93$.

The maximum amplitude $q_{1}^{\max }$ can easily be found from Eq. (7) in the steady-state limit, which yields an implicit equation for the magnitude $\left|q_{1}\right|$ of the steady-state amplitude. ${ }^{14}$ Sweeping the driving frequency, the maximum amplitude is attained when $d\left|q_{1}\right| / d \Omega=0$, which results in the cubic equation

$$
4 \tilde{g}=\left|\tilde{q}_{1}^{\max }\right|\left(4 \tilde{\gamma}+\tilde{\eta}\left|\tilde{q}_{1}^{\max }\right|^{2}\right) .
$$

Here, $\tilde{\gamma}$ and $\tilde{g}$ depend on the bias voltage $V_{\mathrm{dc}}$ via $q_{0}$ and $f_{\text {ac }}$, respectively. However, note that only $\tilde{g}$ depends on the ac voltage. Due to the different dependencies of $q_{0}$ and $\left|q_{1}^{\max }\right|$ on the bias voltage, one can achieve a crossover from NLD- to LD-dominated behavior by increasing the bias voltage. This is shown in Fig. 3(a). In the limit of small $V_{\mathrm{dc}},\left|\tilde{q}_{1}^{\max }\right| \approx$ $(4 \tilde{g} / \tilde{\eta})^{1 / 3} \propto V_{\mathrm{ac}}^{1 / 3} V_{\mathrm{dc}}^{1 / 3}$ and $\tilde{\delta}>1$, i.e., NLD dominates. For large $V_{\mathrm{dc}},\left|\tilde{q}_{1}^{\max }\right| \approx \tilde{g} / \tilde{\gamma} \propto V_{\mathrm{ac}} V_{\mathrm{dc}}^{-3}$ and $\tilde{\delta}$ goes to zero with increasing $V_{\mathrm{dc}}$. Since the static displacement is determined only by the geometry and the bias voltage, and the maximal amplitude additionally depends on the ac voltage, the crossover can also be realized by tuning $V_{\text {ac }}$, which is shown in Fig. 3(b). Equating the expressions for $\left|\tilde{q}_{1}^{\max }\right|$ in the two limits gives an estimate for the crossover for both voltages. Additionally, due to the dependencies of $q_{0} \propto T_{0}^{-1}$ and $\Omega_{0} \propto \sqrt{T_{0}}$ on the initial tension $T_{0}$, one finds that the damping ratio $\tilde{\delta}$ increases with increasing tension in both regimes $\left(\tilde{\delta} \propto T_{0}^{3}\right.$ and $\tilde{\delta} \propto T_{0}$ in the LD and NLD regimes, respectively). Thus, the nonlinear damping is enhanced for larger $T_{0}$.

\section{Quality factor}

To quantify the energy loss, we consider the quality factor $Q=\Omega_{0}\left\langle E_{\perp}\right\rangle /\left\langle\dot{E}_{\perp}\right\rangle$, which measures the time-averaged dissipated energy $\left\langle\dot{E}_{\perp}\right\rangle$ normalized to the average energy $\left\langle E_{\perp}\right\rangle$ in the flexural modes. The nonlinearities render $Q$ amplitude dependent. To get a worst-case estimate, we use the maximal amplitude. In the slow envelope approximation, we find

$$
\frac{1}{Q} \approx \frac{\Omega_{0}\left(\gamma+\frac{1}{4} \eta\left|q_{1}^{\max }\right|^{2}\right)}{m \Omega_{0}^{2}+\frac{1}{2} \frac{3}{8} \alpha\left|q_{1}^{\max }\right|^{2}} .
$$

The nature of the damping influences $Q$. In the LD-dominated regime $\tilde{\delta} \ll 1, Q$ is independent of the vibrational amplitude $Q_{\mathrm{LD}} \approx m \Omega_{0} / \gamma$. In contrast, for $\tilde{\delta}>1$, one gets $Q_{\mathrm{NLD}} \approx$ $4 m \Omega_{0} /\left(\eta\left|q_{1}^{\max }\right|^{2}\right)$ for $\tilde{\eta}>1$. Thus, $Q$ increases with decreasing driving strength. This agrees with the conclusions of Ref. 4.

Figure 4(a) shows the quality factor as a function of bias voltage for constant $V_{\mathrm{ac}}$. As expected, $Q$ decreases with increasing bias and excitation voltages and its behavior with regard to applied voltage changes qualitatively at the crossover between LD and NLD regimes. The asymptotic LD behavior limits the maximally attainable $Q$ factor, which is indicated by the gray area. We also compare to the case where the LD is additionally caused by a mechanism that does not depend on the bias voltage leading to $Q_{0}$. In this case, the effective $Q$ factor $Q_{\text {eff }}^{-1}=Q^{-1}+Q_{0}^{-1}$ has a cutoff for small $V_{\mathrm{dc}}$ as shown in Fig. 4(b), which further limits the region of attainable $Q$ factors. The qualitative difference between LD and NLD is still present and should be experimentally observable. Most importantly, by decreasing $V_{\text {ac }}$, the maximally attainable $Q$ factor, which is determined by other damping mechanisms, can be approached.
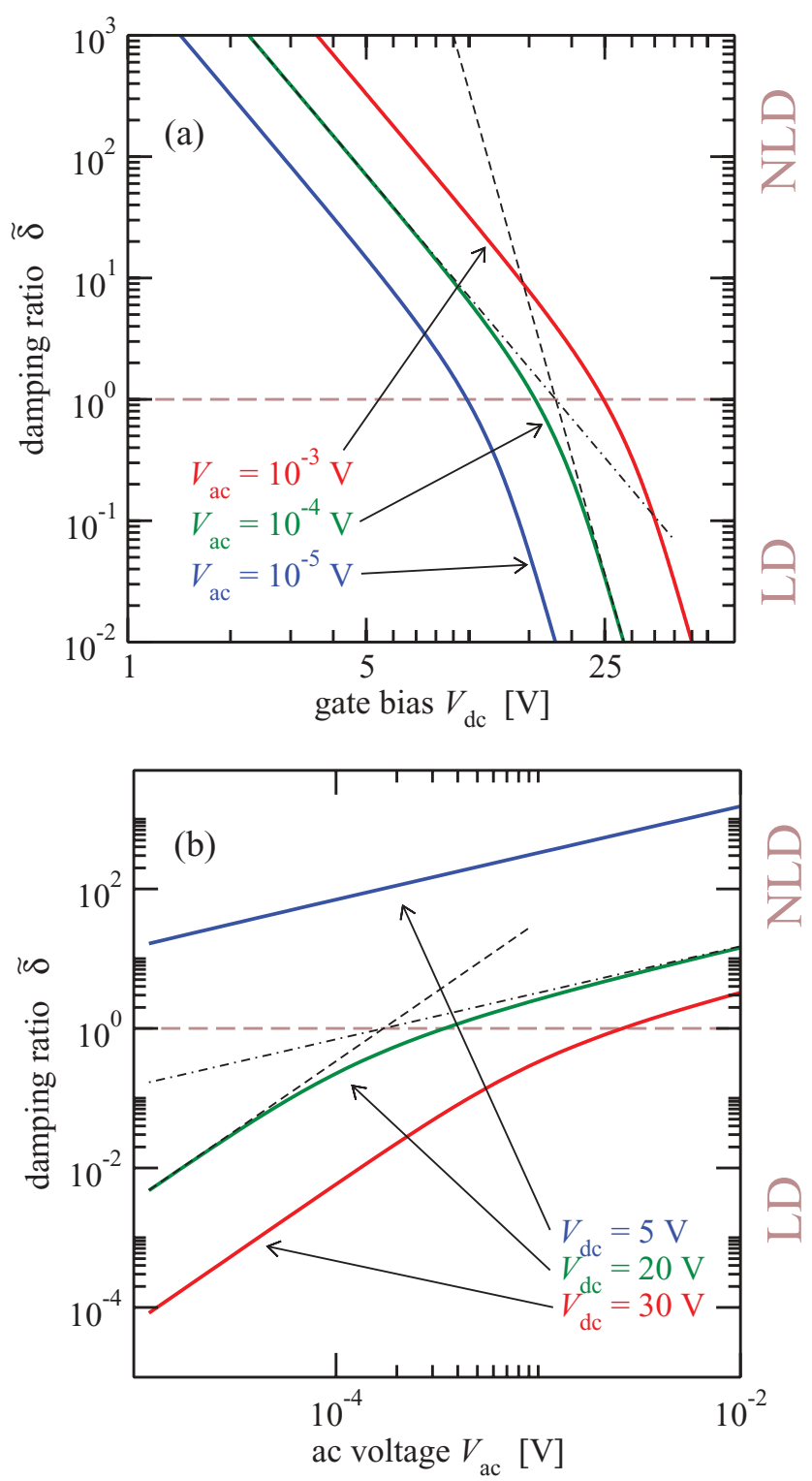

FIG. 3. (Color online) Ratio $\tilde{\delta}$ of nonlinear (NLD) and linear (LD) damping terms according to Eq. (9); (a) bias voltage and (b) ac voltage dependence. The thin dashed and dashed-dotted lines show the asymptotic behavior for strong LD and NLD. A crossover between the two regimes is achieved by changing the bias voltage. Parameters are given in Table I. 

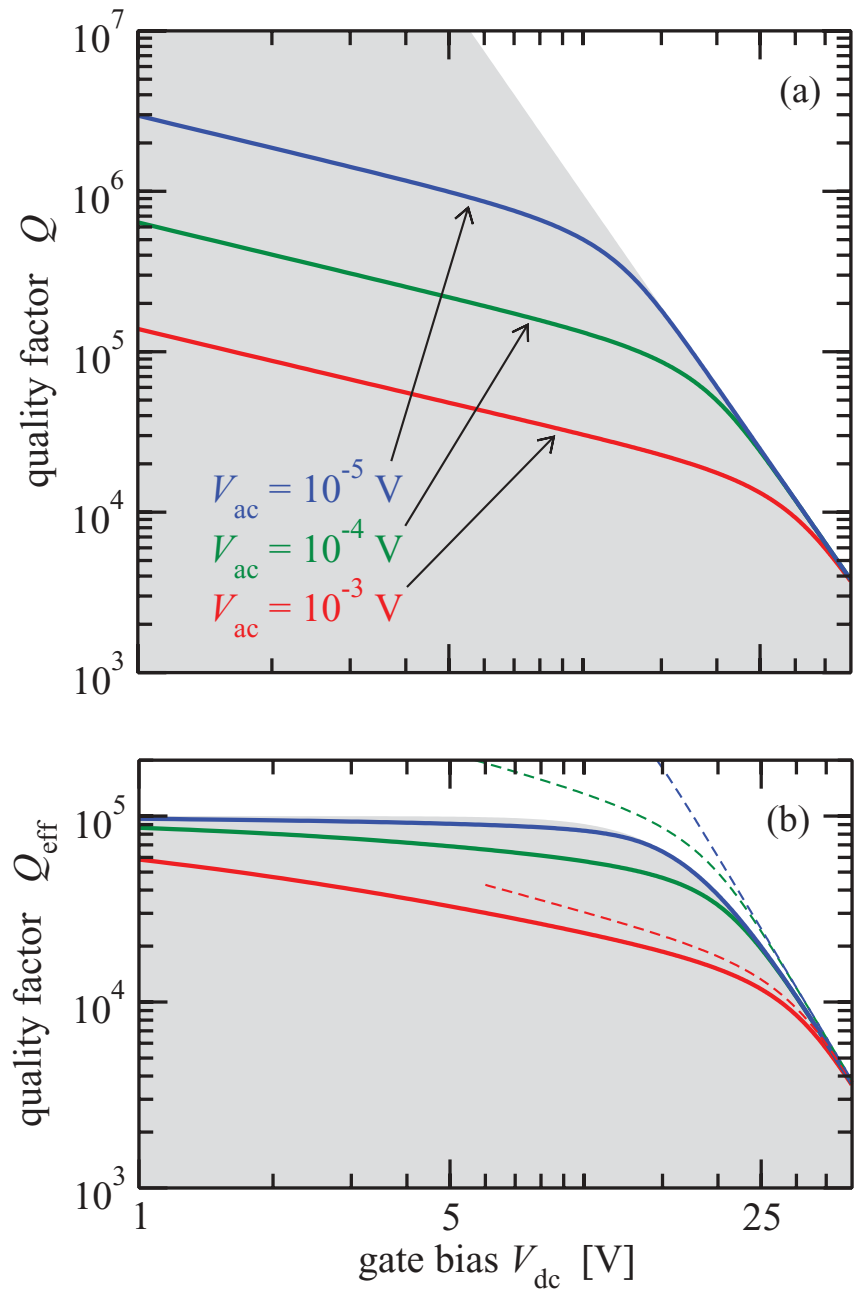

FIG. 4. (Color online) Quality factor $Q$ vs bias voltage. (a) $Q$ calculated from Eq. (20) and (b) with additional voltage-independent damping $Q_{\text {eff }}^{-1}=Q^{-1}+Q_{0}^{-1}$ with $Q_{0}=10^{5}$. The gray area indicates the region of attainable $Q$ factors. The dashed lines correspond to the behavior in (a). Parameters are given in Table I.

\section{CONCLUSIONS}

In conclusion, we have studied coupling between flexural vibrations and in-plane displacements as a physical mechanism for damping of flexural modes in graphene resonators. A characteristic consequence, which influences the behavior of the dependence of the quality factor on bias and excitation voltages, is the competition between static deflection and vibrational amplitude. We note that the same type of behavior would naturally occur for any dissipative process which couples linearly to the strain; for example, Ohmic dissipation induced by synthetic gauge fields. ${ }^{19}$ The crossover should allow for an experimental verification of this class of damping mechanisms.

\section{ACKNOWLEDGMENTS}

We thank J. Atalaya for helpful discussions. The research leading to these results has received funding (D.M., A.I.) from the EU 7th framework program (FP7/2007-2013) RODIN
(Grant Agreement No. 246026) and the Swedish Research Council (J.K.).

\section{APPENDIX A: RESPONSE OF AN ELASTIC HALF-SPACE}

The displacement response at the surface of an elastic halfspace to a stress acting on the surface is given in terms of a response function by Eq. (3). If the stress is directed parallel to the $x$ axis, the spatial Fourier transform of Eq. (3) reads as

$$
u_{\mathrm{S}}(\vec{k}, z=0, \omega)=-R_{x x}(\vec{k}, \omega) \sigma_{x z}(\vec{k}, \omega),
$$

where $k=\left(k_{x}, k_{y}\right)$ is the surface wave vector in the surface. The response function $R_{x x}(\vec{k}, \omega)$ for finite frequencies is explicitly given by ${ }^{30,31}$

$$
R_{x x}(\vec{k}, \omega)=-\frac{i}{\rho_{\mathrm{S}} c_{\mathrm{T}}^{2}}\left(\frac{p_{\mathrm{T}}(\omega, k)}{S(\omega, k)} \frac{\omega^{2}}{c_{\mathrm{T}}^{2}} \frac{k_{x}^{2}}{k^{2}}+\frac{1}{p_{\mathrm{T}}(\omega, k)} \frac{k_{y}^{2}}{k^{2}}\right)
$$

with

$$
\begin{aligned}
p_{\mathrm{L}, \mathrm{T}}(\omega, k) & =\sqrt{\left(\frac{\omega}{c_{\mathrm{L}, \mathrm{T}}}\right)^{2}+i \varepsilon-k^{2}}, \\
S(\omega, k) & =\left[\left(\frac{\omega}{c_{\mathrm{L}, \mathrm{T}}}\right)^{2}-2 k^{2}\right]^{2}+4 k^{2} p_{\mathrm{L}}(\omega, k) p_{\mathrm{T}}(\omega, k),
\end{aligned}
$$

where $c_{\mathrm{L}}$ and $c_{\mathrm{T}}$ are the longitudinal and transversal speeds of sound, respectively, and the infinitesimal $\varepsilon>0$ ensures causality. Notice that $p_{\mathrm{L}, \mathrm{T}}$ and $S(\omega, k)$ depend only on the modulus $k$ of the wave vector $\vec{k}$. The response function in real space is then

$$
\begin{aligned}
R_{x x}(\vec{x}, \omega) & =\int d^{2} k R_{x x}(\vec{k}, \omega) e^{i \vec{k} \cdot \vec{x}} \\
& =-\frac{2 \pi i}{\rho_{\mathrm{S}} c_{\mathrm{T}}^{2}}\left(\frac{\partial}{\partial x} I_{x}(x, y)+\frac{\partial}{\partial y} I_{y}(x, y)\right) .
\end{aligned}
$$

Here, we defined

$$
\begin{aligned}
& I_{x}(x, y)=\frac{x}{\sqrt{x^{2}+y^{2}}}\left(\frac{\omega}{c_{\mathrm{T}}}\right)^{2} \int d k \frac{p_{\mathrm{T}}(\omega, k)}{S(\omega, k)} J_{1}\left(k \sqrt{x^{2}+y^{2}}\right), \\
& I_{y}(x, y)=\frac{y}{\sqrt{x^{2}+y^{2}}} \int d k \frac{1}{p_{\mathrm{T}}(\omega, k)} J_{1}\left(k \sqrt{x^{2}+y^{2}}\right), \quad(\mathrm{A} 3 \mathrm{c})
\end{aligned}
$$

where $J_{1}$ is a first-order Bessel function of the first kind. Note that

$$
I_{x}(x,-y)=I_{x}(x, y), \quad I_{y}(x,-y)=-I_{y}(x, y) .
$$

The expressions given in Eqs. (A3) are a very convenient starting point for the numerical evaluation of the response function used in Sec. II C.

The zero-frequency response can be directly calculated in real space. ${ }^{24}$ One finds

$$
R_{x x}(\vec{x}, \omega=0)=\frac{1}{4 \pi \rho_{\mathrm{S}} c_{\mathrm{T}}^{2}} \frac{2\left(c_{\mathrm{T}}^{2}-c_{\mathrm{L}}^{2}\right) x^{2}-c_{\mathrm{L}}^{2} y^{2}}{\left(c_{\mathrm{L}}^{2}-c_{\mathrm{T}}^{2}\right)\left(x^{2}+y^{2}\right)^{3 / 2}} .
$$




\section{APPENDIX B: STATIC DISPLACEMENT}

In the static limit, the equations for the in-plane and out-ofplane displacements (4) within the suspended region become

$$
\begin{aligned}
T_{1} u_{, x x}+\frac{T_{1}}{2} \partial_{x}\left(w_{, x}^{2}\right) & =0, \\
-\frac{T_{1}}{2} \partial_{x}\left[\left(2 u_{, x}+w_{, x}^{2}\right) w_{, x}\right] & =f_{\mathrm{dc}},
\end{aligned}
$$

with vanishing boundary conditions at $x= \pm \ell / 2$ for the outof-plane displacement. To find the proper boundary conditions for the in-plane displacement, we need to consider the coupling to the substrate in the nonsuspended region. Here, the equation for the in-plane displacement (4b) is given by

$$
T_{1} u_{, x x}-K(x)\left[u(x)-\bar{u}_{S} / b\right]=0 .
$$

Following the same line of reasoning as in the main text, the static substrate response can be written as

$$
\bar{u}_{\mathrm{S}}(x)=-\int_{-L / 2}^{L / 2} \frac{d x^{\prime}}{(2 \pi)^{2}} \bar{R}_{x x}\left(x-x^{\prime}\right) \Theta\left(\left|x^{\prime}\right|-\ell / 2\right) h\left(x^{\prime}\right)
$$

with $h(x)=K_{0}\left(u-\bar{u}_{S} / b\right)$ and $\bar{R}_{x x}\left(x-x^{\prime}\right)$ being the static response function for an elastic half-space given by Eq. (A5) integrated over $y$. To treat the problem analytically, we convert Eqs. (B2) and (B3) into a local equation for the in-plane displacement. In the limit of very strong coupling to the substrate, the spatial variation of $h(x)$ is small, in which case

$$
\begin{aligned}
& -\int_{-L / 2}^{L / 2} \frac{d x^{\prime}}{(2 \pi)^{2}} \bar{R}_{x x}\left(x-x^{\prime}\right) \Theta\left(\left|x^{\prime}\right|-\ell / 2\right) h\left(x^{\prime}\right) \\
& \approx-h(x) \int_{-L / 2}^{L / 2} \frac{d x^{\prime}}{(2 \pi)^{2}} \bar{R}_{x x}\left(x-x^{\prime}\right) \Theta\left(\left|x^{\prime}\right|-\ell / 2\right) .
\end{aligned}
$$

This makes it possible to solve for $h(x)$ in terms of the in-plane displacement $u(x)$. One finds

$$
h(x)=\frac{K_{0}}{1-\bar{R}_{0}(x) K_{0}} u(x),
$$

where $\bar{R}_{0}(x) \equiv(2 \pi)^{-2} \int_{-L / 2}^{L / 2} d x^{\prime} \bar{R}_{x x}\left(x-x^{\prime}\right) \Theta\left(\left|x^{\prime}\right|-\ell / 2\right)$. This expression is valid outside the suspended region and is approximately given by $h(x) \approx-1 / \bar{R}_{0}(x)$, which assumes $K_{0} \bar{R}_{0}(x) \gg 1$. Consequently, the equation for the in-plane displacement, Eq. (B2), is modified to become

$$
T_{1} u_{, x x}+\bar{R}_{0}(x)^{-1} u=0
$$

for $|x|>\ell / 2$. Thus, the effect of the substrate is reduced to that of a spring with a spatially varying spring constant. The displacement $u$ is expected to decay exponentially to zero in the clamped region with a decay length $\lambda \equiv \sqrt{\bar{R}_{0}(x) T_{1}}$. For the substrate parameters given in Table I, this amounts to $\lambda \approx 100 \mathrm{~nm}$. As a consequence, within a distance of $100 \mathrm{~nm}$ from the edge of the suspended region, the in-plane displacement $u(x)$ is essentially zero. To a good approximation, we therefore assume vanishing boundary conditions for in-plane displacement at $|x|=\ell / 2$.

Setting $u(x)=\left(T_{0} / T_{1}\right) x+\Delta u(x)$, where the first terms account for initial strain in the graphene, the boundary conditions are $w(x= \pm \ell / 2)=0$ and $\Delta u(x= \pm \ell / 2)=0$.

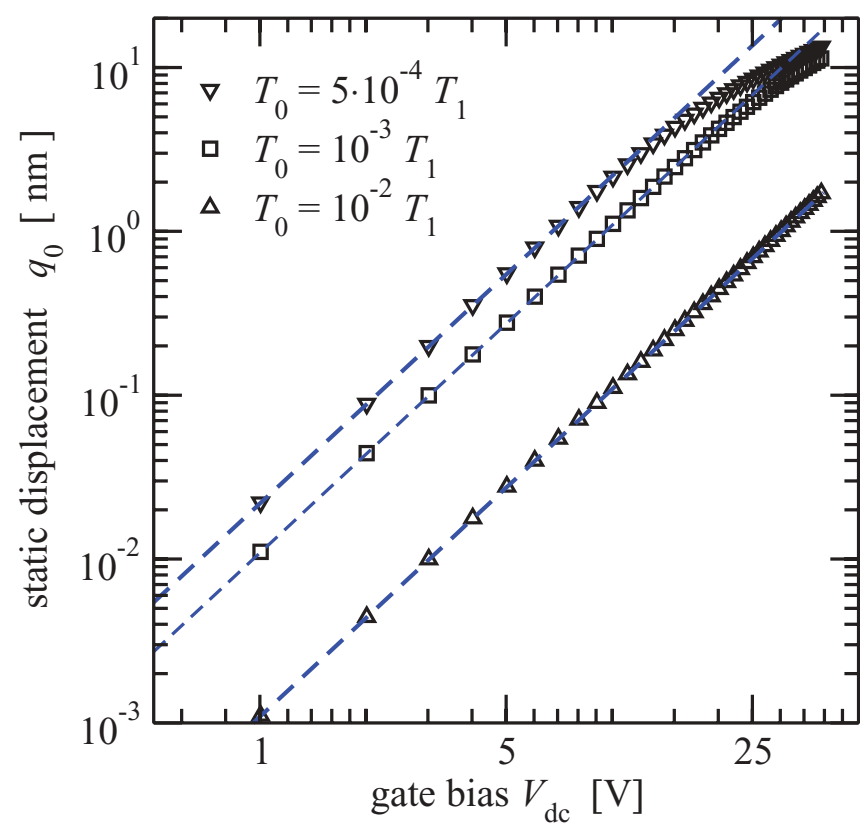

FIG. 5. (Color online) Static deflection $q_{0}$ vs bias voltage for three values of initial tension. The linear approximation [Eq. (B10)] is shown as dashed lines in the figure, while the squares and triangles correspond to the full numerical solution of the static problem.

Using the ansatz $w(x)=q_{0} \phi(x)$ with $\phi(x)=\sqrt{2} \cos \pi x / \ell$, the in-plane equation (B1a) reads as

$$
\Delta u_{, x x}=-\frac{q_{0}^{2}}{2} \partial_{x}\left(\phi_{, x}^{2}\right)
$$

Consequently, the in-plane displacement will be given by

$$
\Delta u(x)=-q_{0}^{2} \frac{\pi^{2}}{\ell^{2}} \int_{0}^{x} d x^{\prime} \sin ^{2} \pi x^{\prime} / \ell+\frac{\pi^{2}}{2 \ell^{2}} q_{0}^{2} x .
$$

By inserting this expression into Eq. (B1b), we obtain

$$
q_{0}\left(\frac{\pi^{2}}{\ell^{2}} T_{0}+\frac{\pi^{4}}{2 \ell^{4}} T_{1} q_{0}^{2}\right)=\frac{2 \sqrt{2}}{\pi} f_{\mathrm{dc}} .
$$

This is a purely algebraic equation for the static deflection. In the limit $q_{0} \ll \frac{\ell}{\pi} \sqrt{\frac{T_{0}}{T_{1}}} \approx 10 \mathrm{~nm}$ for $\ell=1 \mu \mathrm{m}$ and $T_{0} / T_{1}=$ $10^{-3}$, the cubic term can be neglected and $q_{0} \propto f_{\mathrm{dc}}$.

To compute $q_{0}$, we need to consider the electrostatic interaction with the back gate. The static force acting on the graphene is given by Eq. (15). Considering the limit $q_{0} \ll d$, we obtain for the static displacement

$$
q_{0}=-\sqrt{2} \frac{\ell^{2} \epsilon_{0} V_{\mathrm{dc}}^{2}}{\pi^{3} T_{0} d^{2}}
$$

which is the expression given in Sec. III. In Fig. 5, the linear approximation (dashed line), given by Eq. (B10), is compared to the full numerical solution of Eq. (B1) (squares and triangles), which takes the substrate into account. The linear approximation remains valid in the displayed interval for the two larger values of initial strain $T_{0} / T_{1}$, while a more 
significant deviation is apparent for the lowest value of the strain.

\section{APPENDIX C: INFLUENCE OF TUNING AND INITIAL TENSION ON THE QUALITY FACTOR}

In Sec. III A, we discussed the voltage dependence of the resonance frequency (tuning curve) and showed that the frequency can be substantially tuned by changing the bias voltage $V_{\mathrm{dc}}$. Since the linear and nonlinear damping constants given by Eqs. (8) depend on frequency, the quality factor will also depend on the tuning. In order to quantify the influence of the voltage dependence of the resonance frequency on $Q$, Fig. 6 shows the quality factor for constant $\Omega_{0}=\Omega_{0}(0)$ (dashed lines) and $\Omega_{0}\left(V_{\mathrm{dc}}\right)$ (full lines). One sees that deviations between these two cases appear only for larger voltages $\left(V_{\mathrm{dc}}>20 \mathrm{~V}\right)$. Moreover, the qualitative behavior and the crossover from NLD to LD behavior remains unchanged. This confirms our statement in Sec. III C that the behavior of $Q$ is dominated by the damping coefficients $\gamma$ and $\eta$ rather than the voltage dependence of $\Omega_{0}$.

Additionally, Fig. 6 shows the quality factor for a smaller value of the initial tension. In this case, the quality factor is decreased for all values of the static bias voltage. In the limit of large LD, this is due to the increased static deflection [see Eq. (B10)]. In the opposite limit, the quality factor is independent of the static deflection, and the decrease in quality factor is instead a result of the decreasing resonance frequency $\Omega_{0}(0) \propto \sqrt{T_{0}}$. Furthermore, as argued at the end of

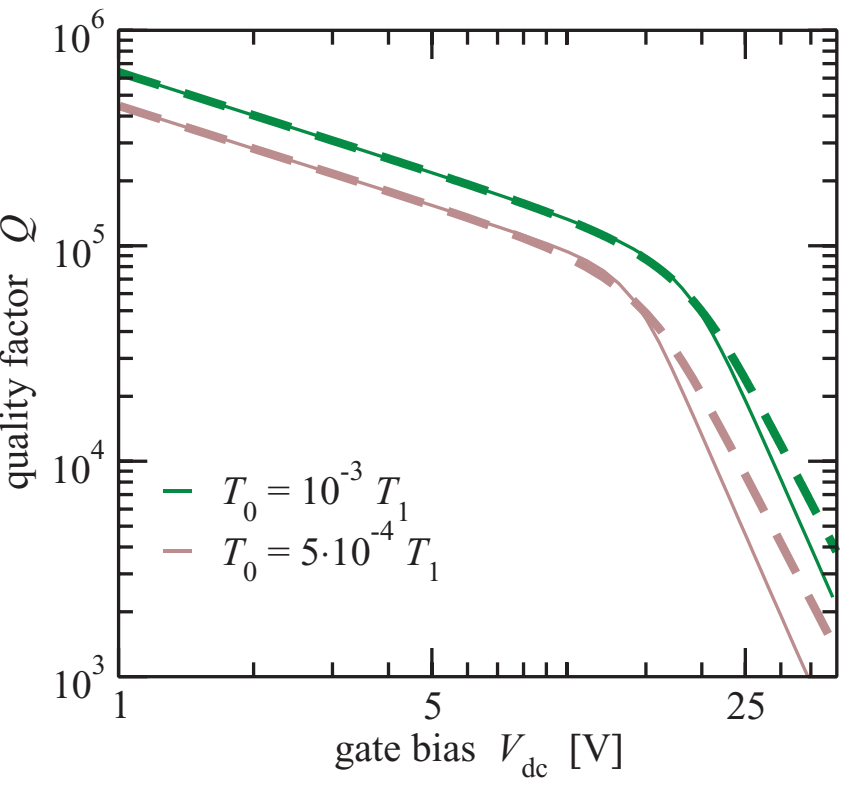

FIG. 6. (Color online) Quality factor $Q$ vs bias voltage calculated from Eq. (20) for $V_{\mathrm{ac}}=10^{-4} \mathrm{~V}$. The full and dashed lines show the result for a voltage dependent $\Omega_{0}\left(V_{\mathrm{dc}}\right)$ and constant $\Omega_{0}=\Omega_{0}(0)$, respectively. Parameters are given in Table I.

Sec. III B, the crossover between NLD and LD is shifted toward lower values of the bias voltage, signifying a decrease in the importance of NLD for lower tension. *alexander.croy@chalmers.se

${ }^{1}$ J. S. Bunch, A. M. van der Zande, S. S. Verbridge, I. W. Frank, D. M. Tanenbaum, J. M. Parpia, H. G. Craighead, and P. L. McEuen, Science 315, 490 (2007).

${ }^{2}$ A. Eriksson, S. Lee, A. A. Sourab, A. Isacsson, R. Kaunisto, J. M. Kinaret, and E. E. B. Campbell, Nano Lett. 8, 1224 (2008).

${ }^{3}$ C. Chen, S. Rosenblatt, K. I. Bolotin, W. Kalb, P. Kim, I. Kymissis, H. L. Stormer, T. F. Heinz, and J. Hone, Nat. Nanotechnol. 4, 861 (2009).

${ }^{4}$ A. Eichler, J. Moser, J. Chaste, M. Zdrojek, I. Wilson-Rae, and A. Bachtold, Nat. Nanotechnol. 6, 339 (2011).

${ }^{5}$ R. Lifshitz and M. L. Roukes, Phys. Rev. B 61, 5600 (2000).

${ }^{6}$ M. C. Cross and R. Lifshitz, Phys. Rev. B 64, 085324 (2001).

${ }^{7}$ I. Wilson-Rae, Phys. Rev. B 77, 245418 (2008).

${ }^{8}$ L. G. Remus, M. P. Blencowe, and Y. Tanaka, Phys. Rev. B 80, 174103 (2009).

${ }^{9}$ C. Seoánez, F. Guinea, and A. H. Castro Neto, Phys. Rev. B 76, 125427 (2007).

${ }^{10}$ A. D. O'Connell, M. Hofheinz, M. Ansmann, R. C. Bialczak, M. Lenander, E. Lucero, M. Neeley, D. Sank, H. Wang, M. Weides, J. Wenner, J. M. Martinis, and A. N. Cleland, Nature (London) 464, 697 (2010).

${ }^{11}$ J. D. Teufel, T. Donner, D. Li, J. W. Harlow, M. S. Allman, K. Cicak, A. J. Sirois, J. D. Whittaker, K. W. Lehnert, and R. W. Simmonds, Nature (London) 475, 359 (2011).

${ }^{12}$ A. Voje, J. M. Kinaret, and A. Isacsson, Phys. Rev. B 85, 205415 (2012).
${ }^{13}$ M. Dykman and M. Krivoglaz, Sov. Sci. Rev., Sect. A 5, 265 (1984)

${ }^{14} \mathrm{R}$. Lifshitz and M. Cross, Nonlinear Dynamics of Nanomechanical and Micromechanical Resonators (Wiley, New York, 2008), Chap. 1 .

${ }^{15}$ S. Zaitsev, O. Shtempluck, E. Buks, and O. Gottlieb, Nonlinear Dynam. 67, 859 (2012).

${ }^{16}$ R. Zwanzig, J. Stat. Phys. 9, 215 (1973).

${ }^{17}$ K. Lindenberg and V. Seshadri, Phys. A (Amsterdam) 109, 483 (1981).

${ }^{18}$ A. H. Castro Neto, F. Guinea, N. M. R. Peres, K. S. Novoselov, and A. K. Geim, Rev. Mod. Phys. 81, 109 (2009).

${ }^{19}$ F. von Oppen, F. Guinea, and E. Mariani, Phys. Rev. B 80, 075420 (2009).

${ }^{20}$ J. Sabio, C. Seoánez, S. Fratini, F. Guinea, A. H. Castro Neto, and F. Sols, Phys. Rev. B 77, 195409 (2008).

${ }^{21}$ S. P. Koenig, N. G. Boddeti, M. L. Dunn, and J. S. Bunch, Nat. Nanotechnol. 6, 543 (2011).

${ }^{22}$ S. Viola Kusminskiy, D. K. Campbell, A. H. Castro Neto, and F. Guinea, Phys. Rev. B 83, 165405 (2011).

${ }^{23}$ Statistical Mechanics of Membranes and Surfaces, edited by D. Nelson, T. Piran, and S. Weinberg (World Scientific, Singapore, 1989).

${ }^{24}$ L. D. Landau and E. M. Lifshitz, Theory of Elasticity, 3rd ed., edited by A. M. Kosevich and L. P. Pitaevskiı̌ (Butterworth-Heinemann, Oxford, 1986).

${ }^{25}$ B. I. Yakobson, C. J. Brabec, and J. Bernholc, Phys. Rev. Lett. 76 2511 (1996). 
${ }^{26}$ A. Fasolino, J. H. Los, and M. I. Katsnelson, Nat. Mater. 6, 858 (2007).

${ }^{27}$ J. Atalaya, A. Isacsson, and J. M. Kinaret, Nano Lett. 8, 4196 (2008).

${ }^{28}$ N. Lindahl, D. Midtvedt, J. Svensson, O. A. Nerushev, N. Lindvall, A. Isacsson, and E. E. B. Campbell, Nano Lett. 12, 3526 (2012).

${ }^{29}$ P. S. Swain and D. Andelman, Langmuir 15, 8902 (1999).

${ }^{30}$ B. N. J. Persson, J. Chem. Phys. 115, 3840 (2001).

${ }^{31}$ A. Maradudin and D. Mills, Ann. Phys. (NY) 100, 262 (1976).
${ }^{32}$ A. W. Barnard, V. Sazonova, A. M. van der Zande, and P. L. McEuen, PNAS 109, 19093 (2012).

${ }^{33}$ We found that $\bar{R}_{x x}\left(x-x^{\prime}, \omega\right)$ is well approximated by the integral $\int_{-b / 2}^{b / 2} d y R_{x x}\left(x-x^{\prime}, y, \omega\right)$.

${ }^{34}$ W. H. Press, B. P. Flannery, S. A. Teukolsky, and W. T. Vetterling, Numerical Recipes in C: The Art of Scientific Computing (Cambridge University Press, Cambridge, 1992), p. 994.

${ }^{35}$ C. Lee, X. Wei, J. W. Kysar, and J. Hone, Science 321, 385 (2008).

${ }^{36}$ B. N. J. Persson and H. Ueba, Europhys. Lett. 91, 56001 (2010). 